\title{
CONTROL DE MALEZAS EN ARROZ DE TEMPORAL CON CLOMAZONE, PROPANIL Y 2,4-D 1
}

\author{
Valentín A. Esqueda
}

\section{RESUMEN}

Control de malezas en arroz de temporal con clomazone, propanil y 2,4-D. Entre los años de 1996 y 1997 se establecieron tres experimentos con el objetivo de evaluar el efecto del herbicida clomazone solo y en mezcla con propanil y 2,4-D en el control de malezas y la toxicidad al arroz cv. Milagro Filipino desarrollado en condiciones de temporal. Los tres experimentos se establecieron en Los Naranjos, Mpio. de Tres Valles en el estado de Veracruz. Las especies dominantes de malezas fueron: Cyperus iria, Echinochloa colona, Cyperus rotundus, Scleria setuloso-ciliata y Malachra fasciata. El clomazone aplicado en preemergencia, controló eficientemente E. colona en dosis de 0,72 y $0,96 \mathrm{~kg}$ i.a./ha, pero sólo tuvo un efecto temporal y limitado sobre $S$. setuloso-ciliata y no tuvo efecto sobre $C$. iria y $C$. rotundus. La mezcla de clomazone + propanil + 2,4-D controló eficientemente E. colona y C. iria a partir de la dosis de $0,60+1,44+0,24 \mathrm{~kg}$ i.a./ha, y tuvo un buen control inicial de $C$. rotundus, pero su efecto sobre esta especie desapareció entre los 45 y 60 días después de la aplicación. Los rendimientos de arroz obtenidos en los tratamientos en donde se aplicó clomazone solo o en mezcla con propanil y 2,4-D, fueron estadísticamente semejantes a los obtenidos en los testigos.

\begin{abstract}
Weed control in upland rice with clomazone, propanil and 2,4-D. Between 1996 and 1997, three experiments were carried out in order to evaluate the effect of the herbicide clomazone, alone and mixed with propanil and 2,4-D on weed control and toxicity to rice cv. Milagro Filipino, growing under upland conditions. All the experiments were established in Los Naranjos, in the Municipality of Tres Valles, in the state of Veracruz. The dominant weed species in the experimental plots were Cyperus iria, Echinochloa colona, Cyperus rotundus, Scleria setuloso-ciliata and Malachra fasciata. Clomazone applied preemergent, efficiently controlled E. colona at 0.72 and $0.96 \mathrm{~kg} \mathrm{a}$. i./ha, but its effect was partial and temporary on S. setuloso-ciliata and it had no effect on $C$. iria and $C$. rotundus. The mixture of clomazone + propanil $+2,4-\mathrm{D}$ efficiently controlled E. colona and C. iria from $0.60+1.44$ $+0.24 \mathrm{~kg}$ a. i./ha and had a good initial control of C. rotundus, but its effect disappeared 45 to 60 days after the application. Rice yields obtained from clomazone treatments, alone or mixed with propanil and 2,4-D were statistically similar to those of the regional controls.
\end{abstract}

\section{INTRODUCCIÓN}

En el cultivo de arroz de temporal en el estado de Veracruz, el control de la maleza se efectúa mediante la aplicación de herbicidas postemergentes con base en el propanil y el 2,4-D. Debido a que el propanil es un herbicida que no tiene acción residual, por lo general es necesario realizar al menos dos aplicaciones de este herbicida durante la etapa crítica de competencia con las malezas (Esqueda y Acosta, 1985).
El empleo de herbicidas de aplicación preemergente se ha visto limitado, debido a que su efectividad está fuertemente condicionada al contenido de humedad y el estado de preparación del suelo. Sin embargo, con la aplicación en postemergencia temprana de herbicidas residuales en mezcla con propanil se tiene la ventaja de que con una sola aplicación se pueden controlar las malezas emergidas, a la vez que se evitan nuevas emergencias de malezas. De esta manera, el propanil se ha mezclado con oxadiazón (Silveira y Aquino, 1983; Esqueda y Acosta, 1985), piperophos (Imeokparia, 1990), tio-

1 Presentado en la XLIV Reunión Anual del PCCMCA, Nicaragua, 1998.

2 Investigador de Malezas y su control. Campo Experimental Cotaxtla. CIRGOC. INIFAP. SAGAR: apdo. postal 429, C.P. 91700 Veracruz, Ver. México. E mail: cecot54@ver1.telmex.net.mx 
bencarbo y pendimetalina (Ríos, 1991; Stauber et al, 1991; Drees, 1996). El clomazone es un herbicida residual que se aplica en preemergencia o en presiembra incorporado y tiene un excelente control de malezas gramíneas y algunas especies de hoja ancha (Thomson, 1993). Este herbicida requiere menor humedad que los herbicidas residuales antes mencionados (Trujillo y Méndez, 1996), lo cual le confiere ventajas cuando el arroz se desarrolla en condiciones donde la humedad no puede ser controlada. Por lo anterior, se considera que una buena alternativa para el control residual de las malezas gramíneas del arroz de temporal podría ser la aplicación de clomazone en preemergencia, o bien si el complejo de malezas está conformado por gramíneas y especies de hoja ancha, la aplicación en postemergencia temprana de la mezcla de clomazone con propanil y 2,4-D amina.

El objetivo de la presente investigación fue determinar el efecto de diferentes dosis de clomazone aplicado en preemergencia, o en postemergencia temprana en mezcla con propanil y $2,4-\mathrm{D}$ en el control de la maleza y la toxicidad al cultivo de arroz de temporal.

\section{MATERIALES Y MÉTODOS}

Se establecieron tres experimentos (uno en 1996 y dos en 1997) en la zona arrocera de Los Naranjos, localizada en el municipio de Tres Valles, en el estado de Veracruz, México. Los tres experimentos se desarrollaron exclusivamente bajo condiciones de temporal. En 1996, en el sitio experimental se cuantificó una precipitación pluvial de $1.214,2 \mathrm{~mm}$ durante el período desde la siembra hasta la cosecha del arroz y en 1997 la precipitación pluvial registrada en este período fue de 1088,1 mm.
Los tratamientos evaluados en cada experimento, dosis, época de aplicación y ciclo agrícola se decriben en el Cuadro 1. En los tres experimentos, los tratamientos estuvieron distribuídos en el terreno de acuerdo a un diseño experimental de bloques completos al azar con cuatro repeticiones. Se utilizó semilla de arroz de la variedad Milagro Filipino, la cual fue sembrada "a chorrillo" a una densidad equivalente a $100 \mathrm{~kg} / \mathrm{ha}$ en el experimento de 1996 y de $75 \mathrm{~kg} / \mathrm{ha}$ en los dos experimentos de 1997. Las parcelas experimentales constaron de diez surcos de $10 \mathrm{~m}$ de longitud y $0,30 \mathrm{~m}$ de separación entre surcos. Los ocho surcos centrales fueron aplicados con los tratamientos correspondientes, dejando sin aplicar los surcos de las orillas a fin de utilizarlos como testigos laterales enhierbados. Los herbicidas fueron aplicados con una aspersora motorizada de mochila, equipada con cuatro boquillas de abanico plano. El volumen de agua aplicado fue equivalente a $330 \mathrm{l} / \mathrm{ha}$ en el experimento de $1996 \mathrm{y}$ 220 1/ha en los experimentos 1997. Los tratamientos del uno al cuatro se aplicaron en preemergencia al arroz y las malezas contando el terreno con muy buena humedad; los tratamientos del cinco al siete se aplicaron a los ocho días después de la emergencia del arroz (DDE), cuando éste y la mayoría de los zacates tenían un desarrollo de entre dos y cuatro hojas y las especies de malezas de hoja ancha contaban con las primeras dos hojas verdaderas. El tratamiento ocho consistió en dos aplicaciones; en la primera, se aplicó la mezcla de propanil + 2,4-D y fue efectuada a los $15 \mathrm{DDE}$, cuando el arroz tenía entre cuatro y cinco hojas, los zacates entre cuatro y seis hojas y las hojas anchas cuatro hojas verdaderas. Para la segunda aplicación se utilizó únicamente propanil a los 29 DDE, para controlar una nueva emergencia de zacates. Las parcelas correspondientes a los testigos limpios fueron deshierbadas manualmente o con azadón a los 12, 20, 32 y 69 DDE. En las parcelas de los testigos

Cuadro 1. Tratamientos de los experimentos de evaluación del herbicida clomazone, sólo y en mezcla con propanil y 2,4-D en arroz de temporal. Campo Experimental Cotaxtla. CIRGOC. INIFAP. SAGAR.

\begin{tabular}{|c|c|c|c|c|c|}
\hline No. & Tratamiento & $\begin{array}{c}\text { Dosis } \\
\text { (kg i.a./ha) }\end{array}$ & $\begin{array}{c}\text { Epoca de aplicación } \\
\text { No. }\end{array}$ & $\begin{array}{l}\text { Experimento } \\
\text { agrícola }\end{array}$ & Ciclo \\
\hline 1 & Clomazone & 0,48 & PRE & 2 & 1997 \\
\hline 2 & Clomazone & 0,72 & PRE & $1-2$ & $1996-1997$ \\
\hline 3 & Clomazone & 0,96 & PRE & 2 & 1997 \\
\hline 4 & Pendimetalina $(*)$ & 1,58 & PRE & 2 & 1997 \\
\hline 5 & Clomazone + Propanil $+2,4-\mathrm{D}$ & $0,48+1,44+0,24$ & $8 \mathrm{DDE}$ & $1-3$ & $1996-1997$ \\
\hline 6 & Clomazone + Propanil $+2,4-\mathrm{D}$ & $0,60+1,44+0,24$ & $8 \mathrm{DDE}$ & $1-3$ & $1996-1997$ \\
\hline 7 & Clomazone + Propanil $+2,4-\mathrm{D}$ & $0,72+1,44+0,24$ & $8 \mathrm{DDE}$ & 3 & 1997 \\
\hline 8 & Propanil $+2,4-\mathrm{D} /$ Propanil $(*)$ & $2,88+0,48 / 2,88$ & $15 \mathrm{DDE} / 29 \mathrm{DDE}$ & $1-3$ & $1996-1997$ \\
\hline 9 & Testigo Limpio & & & 1 & 1996 \\
\hline $\begin{array}{l}10 \\
(*)\end{array}$ & $\begin{array}{l}\text { Testigo Enhierbado } \\
\text { tigos regionales }\end{array}$ & & & $1-2-3$ & $1996-1997$ \\
\hline
\end{tabular}

A los tratamientos del cinco al ocho se les agregó el surfactante LI700 en dosis de 1,5 ml por litro de agua utilizada. 
enhierbados se permitió el libre desarrollo de las malezas durante todo el ciclo del arroz.

La densidad de población de malezas se determinó utilizando cuadrantes de 1 x 1 m (1996) ó 0,5 x 0,5 m (1997), lanzados al azar en las parcelas correspondientes a los testigos enhierbados. Los conteos se llevaron a cabo a los 15 y 30 días después de aplicados los tratamientos (DDA).

Las evaluaciones de control de las especies de malezas dominantes se efectuaron en forma visual, utilizando la escala de cero a $100 \%$, en donde 0 significó que la maleza no fue afectada y $100 \%$ que fue completamente destruída. Las evaluaciones se llevaron a cabo los 15, 30, 45 y 60 DDA. Paralelamente a las evaluaciones de control de malezas, se evaluó visualmente la toxicidad al arroz; los síntomas se describieron y se asignaron valores en la escala de cero a $100 \%$, en donde cero significó que el arroz no fue afectado y $100 \%$ que fue completamente destruído.

Al tiempo de la cosecha se determinó el rendimiento de arroz palay al $14 \%$ de humedad y se efectuaron análisis de varianza para este factor. Como prueba de separación de medias se utilizó Tukey 0,05.

\section{RESULTADOS Y DISCUSIÓN}

En los lotes experimentales se presentaron diez especies de malezas, pertenecientes a cinco familias botánicas. La densidad de población de malezas fluctuó entre 7'210,000 y 12'790,000 plantas/ha a los 15 DDA y 6'630,000 y 15'290,000 plantas/ha a los 30 DDA. Las especies dominantes fueron: Cyperus iria, Echinochloa colona y $C$. esculentus, que en conjunto representaron entre el 88,4 y $97 \%$ de la población total de malezas en las diferentes épocas de evaluación (Cuadro 2).

El clomazone aplicado en preemergencia no tuvo efecto alguno sobre $C$. iria. Pendimetalina en preemergencia tuvo un efecto temporal sobre esta especie, ya que mientras que a los 15 DDA, este herbicida mostraba controles de $97 \%$, a los 45 DDA, su control se redujo a $30 \%$ y su efecto se había perdido completamente a los 60 DDA. A los 15 DDA, los tratamientos en que el clomazone se aplicó en mezcla con propanil y 2,4-D mostraron controles totales o casi totales de $C$. iria, los cuales disminuyeron ligeramente en las siguientes fechas de evaluación hasta terminar con controles de entre 75 y $88 \%$ a los 60 DDA. La mezcla de propanil + 2,4-D seguida de propanil mostró un control casi total de $C$. iria a los 15 DDA y éste se mantuvo por arriba del $90 \%$ hasta los 60 DDA (Cuadro 3).

A los 15 DDA, el control de E. colona por el clomazone aplicado en preemergencia fluctuó entre $85 \%$ con la dosis de $0,48 \mathrm{~kg} / \mathrm{ha}$ y $99 \%$ con la dosis de $0,96 \mathrm{~kg} / \mathrm{ha}$. A los 60 DDA, este herbicida a las dosis de 0,72 y 0,96 $\mathrm{kg} /$ ha todavía mostraba controles de E. colona superiores al $90 \%$, mientras que con la dosis de $0.48 \mathrm{~kg} / \mathrm{ha}$ el control se redujo al 79\%. A su vez, en las diferentes épocas de evaluación, el efecto de pendimetalina sobre esta maleza, fue muy similar al obtenido con la dosis más baja de clomazone. Las mezclas de clomazone con propanil y 2,4-D tuvieron un control de E. colona de entre 89 y $94 \%$ a los 15 DDA. Con las dosis intermedia y alta de esta mezcla, el efecto sobre esta maleza se mantuvo o disminuyó ligeramente en las siguientes épocas de

Cuadro 2. Especies y densidad de población de malezas (miles/ha) presentes en los lotes experimentales a los 15 y 30 DDA. Campo Experimental Cotaxtla. CIRGOC. INIFAP. SAGAR.

\begin{tabular}{|c|c|c|c|c|c|c|c|c|}
\hline \multirow[b]{2}{*}{ Nombre científico } & \multirow[b]{2}{*}{ Familia } & \multicolumn{3}{|c|}{ Experimento 11996} & \multicolumn{2}{|c|}{ Experimento 21997} & \multicolumn{2}{|c|}{ Experimento 31997} \\
\hline & & $\begin{array}{l}\text { Ciclo de } \\
\text { vida }\end{array}$ & 15 DDA & 30 DDA & 15 DDA & 30 DDA & 15 DDA & 30 DDA \\
\hline Cyperus iria $\mathrm{L}$. & Cyperaceae & Anual & 2,930 & 2,480 & 7,920 & 12,790 & 11,320 & 12,140 \\
\hline Echinochloa colona (L.) Link & Gramineae & Anual & 1,840 & 2,150 & 1,570 & 1,340 & 910 & 550 \\
\hline Cyperus esculentus L. & Cyperaceae & Perenne & 1,860 & 1,320 & $\mathrm{x}$ & $\mathrm{x}$ & $\mathrm{x}$ & $\mathrm{x}$ \\
\hline Scleria setuloso-ciliata Boeck. & Cyperaceae & Anual & 270 & 330 & $\mathrm{x}$ & 70 & 210 & 100 \\
\hline Aeschynomene americana $\mathrm{L}$. & Leguminosae & Anual & 230 & 260 & 420 & 390 & 20 & 30 \\
\hline Malachra fasciata Jacq. & Malvaceae & Anual & 80 & 40 & 360 & 210 & 170 & 170 \\
\hline Panicum fasciculatum Sw. & Gramineae & Anual & $\mathrm{x}$ & 50 & $\mathrm{x}$ & $\mathrm{x}$ & $\mathrm{x}$ & 20 \\
\hline Cyperus rotundus $\mathrm{L}$. & Cyperaceae & Perenne & $\mathrm{x}$ & $\mathrm{x}$ & 460 & 490 & 130 & 50 \\
\hline Caperonia palustris (L.) St. Hil. & Euphorbiaceae & Anual & $\mathrm{x}$ & $\mathrm{x}$ & $\mathrm{x}$ & $\mathrm{x}$ & 10 & 20 \\
\hline Mimosa pudica L. & Leguminosae & Anual & $\mathrm{x}$ & $\mathrm{x}$ & $\mathrm{x}$ & $\mathrm{x}$ & 20 & $\mathrm{x}$ \\
\hline Total & & & 7,210 & 6,630 & 10,730 & 15,290 & 12,790 & 13,080 \\
\hline
\end{tabular}

$\mathrm{x}=$ no se presentó en el área de muestreo. 
Cuadro 3. Efecto de clomazone sólo y en mezcla con propanil y 2,4-D en el control de C. iria, E. colona y C. esculentus a los 15, 30, 45 y 60 días después de la aplicación. Campo Experimental Cotaxtla. CIRGOC. INIFAP. SAGAR.

\begin{tabular}{|c|c|c|c|c|c|}
\hline Tratamiento & $\begin{array}{c}\text { Dosis } \\
\text { (kg i.a./ha) }\end{array}$ & $\begin{array}{c}\text { Epoca de } \\
\text { aplicación }\end{array}$ & $\begin{array}{c}\text { C. iria } \\
15-30-45-60\end{array}$ & $\begin{array}{c}\text { E. colona } \\
15-30-45-60\end{array}$ & $\begin{array}{c}\text { C. esculentus } \\
15-30-45-60\end{array}$ \\
\hline Clomazone $^{1}$ & 0,48 & PRE & $0-0-0-0$ & $85-86-81-79$ & $x-x-x-x$ \\
\hline Clomazone $^{2}$ & 0,72 & PRE & $0-0-0-0$ & $94-93-93-91$ & $x-x-x-x$ \\
\hline Clomazone $^{1}$ & 0,96 & PRE & $0-0-0-0$ & $99-98-98-97$ & $x-x-x-x$ \\
\hline Pendimetalina ${ }^{1}$ & 1,58 & PRE & $97-66-30-0$ & $89-84-79-79$ & $x-x-x-x$ \\
\hline Clomazone + Propanil + 2,4-D & $0,48+1,44+0,24$ & $8 \mathrm{DDE}$ & $98-86-84-75$ & $89-80-78-68$ & $83-89-74-79$ \\
\hline Clomazone + Propanil $+2,4-\mathrm{D}^{2}$ & $0,60+1,44+0,24$ & $8 \mathrm{DDE}$ & $100-94-94-88$ & $94-94-91-88$ & $88-94-84-84$ \\
\hline Clomazone + Propanil + 2,4-D ${ }^{1}$ & $0,72+1,44+0,24$ & $8 \mathrm{DDE}$ & $100-89-86-81$ & $91-91-91-90$ & $x-x-x-x$ \\
\hline Propanil + 2,4-D/ Propanil ${ }^{2}$ & $2,88+0,48 / 2,88$ & $\begin{array}{l}15 \mathrm{DDE} \\
\text { / } 29 \mathrm{DDE}\end{array}$ & $98-99-95-94$ & $97-89-85-85$ & $100-98-97-95$ \\
\hline Testigo Limpio 1 & & & $100-100-100-100$ & $100-100-100-100$ & $100-100-100-100$ \\
\hline Testigo Enhierbado ${ }^{3}$ & & & $0-0-0-0$ & $0-0-0-0$ & $0-0-0-0$ \\
\hline
\end{tabular}

1 Los valores de control fueron obtenidos de un experimento

2 Los valores de control son el promedio de dos experimentos

3 Los valores de control son el promedio de tres experimentos

evaluación, mientras que con la dosis baja, el control se redujo hasta cuantificarse en $68 \%$ a los 60 DDA. La aplicación de propanil + 2,4-D, seguida de propanil controló el $97 \%$ de E. colona a los 15 DDA, el cual disminuyó a $85 \%$ a los 60 DDA (Cuadro 3).

C. esculentus solamente se presentó en el experimento uno (1996), por lo cual no se presentan datos de control para todos los tratamientos. A los 15 DDA, esta especie fue controlada totalmente con la mezcla de propanil + 2,4-D, seguida de propanil, manteniéndose su control igual o superior al 95\% en las siguientes épocas de evaluación. Las mezclas de clomazone + propanil + 2,4-D tuvieron controles iniciales de $C$. iria, de 83 y $88 \%$ cuando se aplicaron 0,48 y $0,60 \mathrm{~kg}$ de clomazone/ha, respectivamente. A los 60 DDA, se observó una ligera reducción en el control de esta especie, terminando su control en 79 y $84 \%$, respectivamente (Cuadro 3).

La emergencia de $S$. setuloso-ciliata comenzó después de los 15 días después de la aplicación de los tratamientos preemergentes, por lo que solamente se reportan los controles obtenidos por estos tratamientos a partir de los 30 DDA. Pendimetalina no mostró efecto alguno sobre $S$. setuloso-ciliata. Clomazone en sus tres dosis ocasionó manchas de color blanco y ligera necrosis en el follaje de S. setuloso-ciliata, sin embargo, estos síntomas desaparecieron completamente entre los 30 y 45 DDA. Las mezclas de clomazone + propanil + 2,4-D mostraron controles de esta maleza de entre 83 y $88 \%$ a los 15 DDA. En las mezclas con las dosis intermedia y alta de clomazone, los controles disminuyeron ligeramente entre una época de evaluación y la siguiente, mientras que en la mezcla con la dosis baja de clomazone, el control final de S. setuloso-ciliata fue de solamante el $50 \%$. Con la mezcla de propanil + 2,4-D, seguida de propanil, el control de esta especie fluctuó de $100 \%$ a los 15 y 30 DDA, a $95 \%$ a los 60 DDA (Cuadro 4).

Ninguno de los tratamientos aplicados en preemergencia tuvo efecto sobre $C$. rotundus. Las tres mezclas de clomazone + propanil + 2,4-D tuvieron controles iniciales de $C$. rotundus de $85 \%$ o superiores, sin embargo su efecto sobre esta maleza se redujo progresivamente hasta perderse completamente entre los 45 y 60 DDA. A su vez, con la mezcla de propanil + 2,4-D seguida por propanil, el control de $C$. rotundus fue de aproximadamente $80 \%$ a los 15 y 30 DDA, para disminuir a $63 \%$ a los 60 DDA (Cuadro 4).

$\mathrm{Al}$ igual que con $C$. rotundus, no se observó ningún efecto herbicida sobre M. fasciata por parte de clomazone o pendimetalina aplicados en preemergencia. A los 15 DDA, todos los tratamientos aplicados en postemergencia mostraban un control total de M. fasciata. En los tres tratamientos de clomazone mezclado con propanil y 2,4-D, se observó una reducción paulatina en el control de esta especie, siendo más acentuada en la mezcla que contenía la dosis de clomazone mas baja. A su vez, con la mezcla de propanil + 2,4-D seguida de propanil, todavía se tenía un control de $M$. fasciata de $90 \%$ a los 60 DDA (Cuadro 4).

Se observó un "blanqueamiento" temporal en algunas áreas del follaje de las plantas de arroz que se aplicaron con clomazone en preemergencia a las dosis de 0,72 y $0,96 \mathrm{~kg} / \mathrm{ha}$ o en postemergencia en mezcla con propanil + 2,4-D. A los 15 DDA, en las aplicaciones en 
Cuadro 4. Efecto de clomazone sólo y en mezcla con propanil y 2,4-D en el control de S. setuloso-ciliata, C. rotundus y M. fasciata a los 15, 30, 45 y 60 días después de la aplicación. Campo Experimental Cotaxtla. CIRGOC. INIFAP. SAGAR.

\begin{tabular}{|c|c|c|c|c|c|}
\hline Tratamiento & $\begin{array}{c}\text { Dosis } \\
\text { (kg i.a./ha) }\end{array}$ & $\begin{array}{l}\text { Época de } \\
\text { aplicación }\end{array}$ & $\begin{array}{c}\text { S. setuloso-ciliata } \\
15-30-45-60\end{array}$ & $\begin{array}{c}\text { C. rotundus } \\
15-30-45-60\end{array}$ & $\begin{array}{c}\text { M. fasciata } \\
15-30-45-60\end{array}$ \\
\hline Clomazone $^{1}$ & 0,48 & PRE & $x-15-0-0$ & $0-0-0-0$ & $0-0-0-0$ \\
\hline Clomazone $^{2}$ & 0,72 & PRE & $x-14-0-0$ & $0-0-0-0$ & $0-0-0-0$ \\
\hline Clomazone $^{1}$ & 0,96 & PRE & $x-25-0-0$ & $0-0-0-0$ & $0-0-0-0$ \\
\hline Pendimetalina $^{1}$ & 1,58 & PRE & $x-0-0-0$ & $0-0-0-0$ & $0-0-0-0$ \\
\hline Clomazone + Propanil $+2,4-\mathrm{D}^{2}$ & $0,48+1,44+0,24$ & $8 \mathrm{DDE}$ & $85-70-66-50$ & $85-50-40-0$ & $100-79-74-61$ \\
\hline Clomazone + Propanil $+2,4-\mathrm{D}^{2}$ & $0,60+1,44+0,24$ & $8 \mathrm{DDE}$ & $88-85-84-81$ & $88-58-55-0$ & $100-92-90-79$ \\
\hline Clomazone + Propanil + 2,4-D ${ }^{1}$ & $0,72+1,44+0,24$ & $8 \mathrm{DDE}$ & $83-83-78-76$ & $86-65-58-0$ & $100-100-91-85$ \\
\hline Propanil + 2,4-D / Propanil ${ }^{2}$ & $2,88+0,48 / 2,88$ & $\begin{array}{l}15 \mathrm{DDE} \\
\text { / } 29 \mathrm{DDE}\end{array}$ & $100-100-97-95$ & $78-79-73-63$ & $100-99-98-90$ \\
\hline Testigo Limpio ${ }^{1}$ & & & $100-100-100-100$ & $100-100-100-100$ & $100-100-100-100$ \\
\hline Testigo Enhierbado ${ }^{3}$ & & & $0-0-0-0$ & $0-0-0-0$ & $0-0-0-0$ \\
\hline
\end{tabular}

1 Los valores de control fueron obtenidos de un experimento.

2 Los valores de control son el promedio de dos experimentos.

3 Los valores de control son el promedio de tres experimentos.

preemergencia, este blanqueamiento ocupaba entre el 11 y el $15 \%$ del follaje de las plantas afectadas. A su vez, en esta misma época de evaluación, en las aplicaciones postemergentes de las mezclas de clomazone + propanil + 2,4-D, el blanqueamiento ocupaba solamente entre el uno y $7 \%$ del área foliar. Todos los síntomas de blanqueamiento desaparecieron entre los 15 y 30 DDA. Pendimetalina y la mezcla de propanil + 2,4-D seguida por propanil, no ocasionaron toxicidad al arroz (Cuadro 5).

En el experimento uno (1996), el mayor rendimiento de arroz palay se obtuvo con el tratamiento de la mezcla de propanil + 2,4-D seguida de propanil con aproximadamente 3.5 ton/ha. Este tratamiento fue estadísticamente semejante al testigo limpio y a los dos tratamientos de mezclas de clomazone con propanil y 2,4D. En las parcelas correspondientes a clomazone $(0.72$ $\mathrm{kg} / \mathrm{ha}$ ) aplicado en preemergencia no hubo producción de arroz, debido al efecto de la fuerte competencia al cultivo por las malezas ciperáceas y de hoja ancha que no fueron controladas con ese tratamiento. De igual manera, no hubo producción de grano en las parcelas del testigo enhierbado (Cuadro 5).

En el experimento dos (1997), los rendimientos de arroz palay obtenidos en los diferentes tratamientos variaron de 1.269 a $2.273 \mathrm{~kg} / \mathrm{ha}$, y no hubo diferencia significativa entre los tratamientos, incluyendo el testigo enhierbado. Tanto clomazone como pendimetalina son herbicidas que controlan principalmente gramíneas y tienen un efecto limitado o nulo sobre las malezas de hoja ancha o ciperáceas. Lo anterior ocasionó que al haberse controlado $E$. colona, se eliminará la dominancia que esta especie ejerce sobre las otras malezas y éstas se desarrollarán sin interferencia, ocasionando competencia al arroz y reduciendo su rendimiento de manera semejante a la reducción observada en el testigo enhierbado (Cuadro 5).

En el experimento tres (1997), el rendimiento de arroz palay mas alto correspondió al tratamiento en que se aplicaron $0, .72 \mathrm{~kg} / \mathrm{ha}$ de clomazone en mezcla con propanil $+2,4-\mathrm{D}$, siendo estadísticamente semejante a los rendimientos obtenidos con las otras dos mezclas de clomazone + propanil + 2,4-D. A su vez, el rendimiento de grano obtenido con la mezcla de propanil + 2,4-D seguida de propanil fue semejante al del testigo enhierbado (Cuadro 5). El bajo rendimiento obtenido con este último tratamiento, pudo deberse a una reducción en el control de malezas (principalmente E. colona), debido a que en la época de aplicación de la segunda parte de este tratamiento, el lote experimental estaba sufriendo un prolongado período de sequía.

El análisis conjunto de los datos experimentales, revela que la mezcla de clomazone + propanil + 2,4-D, puede competir ventajosamente con el tratamiento regional utilizado para el control del complejo de malezas dominantes en los arrozales de temporal en el estado de Veracruz, ya que solamente es necesaria una aplicación para obtener un control de malezas semejante al del testigo regional. 
Cuadro 5. Efecto de los tratamientos en la toxicidad al arroz a los 15, 30, 45 y 60 días después de la aplicación y en el rendimiento de arroz palay al $14 \%$ de humedad. Campo Experimental Cotaxtla. CIRGOC. INIFAP. SAGAR.

\begin{tabular}{|c|c|c|c|c|c|c|c|c|c|}
\hline \multirow[b]{2}{*}{ Tratamiento } & \multirow[b]{2}{*}{$\begin{array}{c}\text { Dosis } \\
\text { (kg i.a./ha) }\end{array}$} & \multirow[b]{2}{*}{$\begin{array}{c}\text { Epoca de } \\
\text { aplicación }\end{array}$} & \multirow{2}{*}{\multicolumn{4}{|c|}{$\begin{array}{c}\text { Toxicidad al arroz } \\
15-30-45-60\end{array}$}} & \multicolumn{3}{|c|}{ Rendimiento (kg/ha) } \\
\hline & & & & & & & $\begin{array}{c}\text { Expto. } 1 \\
\text { (1996) }\end{array}$ & $\begin{array}{c}\text { Expto. } 2 \\
\text { (1997) }\end{array}$ & $\begin{array}{c}\text { Expto. } 3 \\
\text { (1997) }\end{array}$ \\
\hline Clomazone $^{1}$ & 0,48 & PRE & 0 & -0 & -0 & -0 & $\mathrm{x}$ & $2,273 \mathrm{a}$ & $\mathrm{x}$ \\
\hline Clomazone $^{2}$ & 0,72 & PRE & 15 & -0 & -0 & -0 & $0 \mathrm{~b}$ & $2,005 \mathrm{a}$ & $\mathrm{x}$ \\
\hline Clomazone $^{1}$ & 0,96 & PRE & 11 & -0 & -0 & -0 & $\mathrm{x}$ & $1,269 \mathrm{a}$ & $\mathrm{x}$ \\
\hline Pendimetalina $^{1}$ & 1,58 & PRE & 0 & -0 & -0 & -0 & $\mathrm{x}$ & $1,446 \mathrm{a}$ & $\mathrm{x}$ \\
\hline Clomazone + Propanil + 2,4-D ${ }^{2}$ & $0,48+1,44+0,24$ & $8 \mathrm{DDE}$ & 1 & -0 & -0 & -0 & $3,018 \mathrm{a}$ & $\mathrm{x}$ & $2,544 \mathrm{ab}$ \\
\hline Clomazone + Propanil $+2,4-D^{2}$ & $0,60+1,44+0,24$ & $8 \mathrm{DDE}$ & 4 & -0 & -0 & -0 & 3,311 a & $\mathrm{x}$ & 3,367 a \\
\hline Clomazone + Propanil + 2,4-D ${ }^{1}$ & $0,72+1,44+0,24$ & $8 \mathrm{DDE}$ & 7 & -0 & -0 & -0 & $\mathrm{x}$ & $\mathrm{x}$ & 3,417 a \\
\hline Propanil + 2,4-D/ Propanil ${ }^{2}$ & $\begin{array}{l}2,88+0,24 / 2,88 \\
\text { / } 29 \mathrm{DDE}\end{array}$ & $15 \mathrm{DDE}$ & 0 & -0 & -0 & -0 & 3,494 a & $\mathrm{x}$ & $1,504 \mathrm{bc}$ \\
\hline Testigo Limpio $^{1}$ & & & 0 & -0 & -0 & -0 & $3,463 \mathrm{a}$ & $\mathrm{x}$ & $\mathrm{x}$ \\
\hline Testigo Enhierbado 3 & & & 0 & -0 & -0 & -0 & $0 \mathrm{~b}$ & $1,314 \mathrm{a}$ & $514 \mathrm{c}$ \\
\hline
\end{tabular}

Las letras a la derecha de los valores representan la prueba de Tukey $(0,05)$. Valores con la misma letra no son significativamente diferentes entre sí. Las comparaciones son entre tratamientos en cada uno de los experimentos.

1 Los valores de control fueron obtenidos de un experimento.

2 Los valores de control son el promedio de dos experimentos.

3 Los valores de control son el promedio de tres experimentos.

\section{CONCLUSIONES}

Clomazone aplicado en preemergencia controló eficientemente E. colona, a partir de $0,72 \mathrm{~kg} / \mathrm{ha}$, pero no tuvo efecto sobre $C$. iria, C.rotundus y $M$. fasciata y su efecto fue temporal y limitado sobre $S$. setuloso-ciliata.

Pendimetalina tuvo un control de bueno a regular de E. colona, un efecto temporal sobreC. iria y no afectó a C.rotundus, M. fasciata y S. setuloso-ciliata.

La mezcla de clomazone + propanil $+2,4$-D controló eficientemente E. colona, C. iria, C. esculentus, y $M$. fasciata a partir de la dosis de $0,60+1,44+$ $0,24 \mathrm{~kg} / \mathrm{ha}$, pero a esta dosis, el control deS. setulosociliata fue solamente de bueno a regular y su efecto sobre $C$. rotundus se perdió completamente entre los 45 y 60 DDA.

La mezcla de propanil + 2,4-D seguida de propanil tuvo controles eficientes de todas las especies de malezas dominantes, a excepción de C. rotundus, cuyo control fue solamente regular.

El clomazone aplicado solo en dosis de 0,72 y 0,96 $\mathrm{kg} / \mathrm{ha}$ o en mezcla con propanil + 2,4-D causó un ligero "blanqueamiento" en el follaje del arroz, pero éste desapareció entre los 15 y 30 DDA.
Los rendimientos de grano de los tratamientos en que el clomazone se aplicó sólo o en mezcla con propanil + 2,4-D fueron semejantes o superiores a los rendimientos obtenidos en los correspondientes testigos regionales.

\section{LITERATURA CITADA}

DREES, B. M. 1996. Rice production guidelines. Texas Agricultural Extension Service. The Texas A \& M University System. 63 p.

ESQUEDA, E. V. A.; ACOSTA, S. 1985. Daños y control de las malas hierbas en el cultivo de arroz de temporal en el centro del estado de Veracruz y norte de Oaxaca. México, D. F. Secretaría de Agricultura y Recursos Hidráulicos. Instituto Nacional de Investigaciones Agrícolas. Folleto de Investigación Núm. 65. 60 p.

IMEOKPARIA, P. O. 1990. Performance of a new selective rice herbicide in a lowland rice field in Nigeria. Tropical Pest Management 36(4):343-345.

RÍOS, T. A. 1991. Control de malezas en arroz con mezcla física pendimetalín + propanil en la Costa de Nayarit. In: Memorias XII Congreso Nacional de la Ciencia de la Maleza. Asociación Mexicana de la Ciencia de la Maleza. Acapulco, Gro. México. p. 64.

SILVEIRA, F. A.; AQUINO, A. 1983. Weed control and rice production in Brazil. In: Proceedings of the Conference 
on weed control in rice. International Rice Research Institute. International Weed Science Society. Los Baños, Laguna, Philippines, 1981. pp. 133-138.

STAUBER, L. G.; NASTASI, P.;SMITH, R.; BALTAZAR, A.; TALBERT, R. 1991. Barnyardgrass (Echinochloa crus-galli) and bearded sprangletop (Leptochloa fascicularis) control in rice (Oryza sativa). Weed Technology 5(2):337-344.
THOMSON, W. T. 1993. Agricultural Chemicals. Book II Herbicides. Fresno, Thomson Publications. 310 p.

TRUJILLO, O.;MÉNDEZ, D. 1996. Command, inhibidor de pigmentos, en especies de malezas susceptibles. In: Arrocero Moderno, con el mejor entorno ambiental. Serie Agroindustria 2009, Bogotá, Comunicaciones y Asociados Ltda. pp. 123-127. 